\title{
The Impact of Combination Antiretroviral Therapy and its Interruption on Anxiety, Stress, Depression and Quality of Life in Thai Patients
}

\author{
Reto Nüesch $^{*}, 1,2, \S$, Angèle Gayet-Ageron ${ }^{3, \S}$, Ploenchan Chetchotisakd ${ }^{4}$, Wisit Prasithsirikul ${ }^{5}$, \\ Sasisopin Kiertiburanakul ${ }^{6}$, Warangkana Munsakul ${ }^{7}$, Phitsanu Raksakulkarn ${ }^{8}$, Somboon \\ Tansuphasawasdikul ${ }^{9}$, Sineenart Chautrakarn ${ }^{1}$, Kiat Ruxrungtham ${ }^{1}$, Bernard Hirschel ${ }^{3}$, \\ Jintanat Anaworanich ${ }^{1}$ and the STACCATO study group
}

\author{
${ }^{1}$ HIV-NAT, The Thai Red Cross AIDS Research Center, Bangkok, Thailand \\ ${ }^{2}$ Division of Infectious Diseases University Hospital Basel and Hirslanden Clinic St. Anna Lucerne, Switzerland \\ ${ }^{3}$ Division des Maladies infectieuses, Geneva University Hospital, Switzerland \\ ${ }^{4}$ Khon Kaen University, Khon Kaen, Thailand \\ ${ }^{5}$ Bamrasnaradura Institute, Nonthaburi, Thailand \\ ${ }^{6}$ Faculty of Medicine Ramathibodi Hospital, Mahidol University, Bangkok, Thailand \\ ${ }^{7}$ Metropolitan Administration Medical College and Vijira Hospital, Bangkok, Thailand \\ ${ }^{8}$ Sanpatong Hospital, Chiang Mai, Thailand \\ ${ }^{9}$ Buddhachinnaraj Hospital, Phitsanulok, Thailand
}

\begin{abstract}
Objective: Investigation on anxiety, stress, depression, and quality of life (QoL) within STACCATO, a randomised trial of two treatment strategies: CD4 guided scheduled treatment interruption (STI) compared to continuous treatment $(\mathrm{CT})$.

Participants: Thai patients with HIV-infection enrolled in the STACCATO trial.

Methods: Anxiety, depression assessed by the questionnaires Hospital Anxiety and Depression Scale (HADS) and DASS, stress assessed by the Depression Anxiety Stress Scale (DASS), and QoL evaluated by the HIV Medical Outcome Study (MOS-HIV) questionnaires. Answers to questionnaires were evaluated at 4 time-points: baseline, 24 weeks, 48 weeks and at the end of STACCATO.

Results: A total of 251 patients answered the HADS/DASS and 241 answered the MOS-HIV of the 379 Thai patients enrolled into STACCATO (66.2 and 63.6\% respectively). At baseline $16.3 \%$ and $7.2 \%$ of patients reported anxiety and depression using HADS scale. Using the DASS scale, $35.1 \%$ reported mild to moderate and $9.6 \%$ reported severe anxiety; $8.8 \%$ reported mild to moderate and $2.0 \%$ reported severe depression; $42.6 \%$ reported mild to moderate and $4.8 \%$ reported severe stress. We showed a significant improvement of the MHS across time ( $\mathrm{p}=0.001)$, but no difference between arms $(\mathrm{p}=0.17)$. The summarized physical health status score (PHS) did not change during the trial $(\mathrm{p}=0.15)$ nor between arm $(\mathrm{p}=0.45)$. There was no change of MHS or PHS in the STI arm, taking into account the number of STI cycle ( $\mathrm{p}=0.30$ and $0.57)$ but MHS significant increased across time-points $(\mathrm{p}=0.007)$.

Conclusion: Antiretroviral therapy improved mental health and QOL, irrespective of the treatment strategy.
\end{abstract}

Keywords: HIV infection, mental health, quality of life, treatment interruption.

\section{INTRODUCTION}

Combination antiretroviral therapy (cART) has changed HIV from a life threatening into a chronic condition. Anxiety and depression are common features of chronic diseases. Sixteen to $36 \%$ of patients with HIV infection have

*Address correspondence to this author at the Hirslanden-Clinic St. Anna, St. Anna Street 32, CH-6006 Lucerne, Switzerland;

E-mail: reto.nuesch@hirslanden.ch

${ }^{\S}$ Both authors have equally contributed to the manuscript underlying depression [1-3]. Since the advent of cART, few studies have focused on the prevalence of anxiety and depression. They remain common findings affecting the mental health, the quality of life (QoL), and response to cART [4]. Patients with less anxiety are, on average, more educated, employed, have a shorter duration of HIV disease, lower viral load, and more likely of female gender [5]. The perspective of never ending pill ingestion is dispiriting to many patients who feel imprisoned by their chronic disease [6-9]. Drug toxicity and especially lipodystrophy is another well recognized complication of cART that may affect quality of life of treated patients [10-13]. One strategy to 
deal with such problems was to reduce overall drug exposure by scheduled treatment interruptions (STI) but since the publication of the SMART trial's results [14], this strategy is not viewed as an alternative care management anymore [15, 16]. Treatment interruption with subsequent increases in viral load may increase anxiety and affect QoL through symptoms related to increased viremia. Nowadays STI are not recommended as an alternative to continuous antiretroviral therapy. Nevertheless the STACCATO trial substudy on mental disorders and QoL offers an important insight on the evolution of mental health and QOL with continuous and interrupted cART in a resource limited setting.

\section{METHODS}

\section{The Swiss Thai Australia Treatment Interruption Trial (STACCATO)}

STACCATO was a prospective, open-label, randomized, multicentre trial conducted in Thailand, Switzerland and Australia from January 2002 until November 2005 [17]. Patients with chronic HIV-1 infection who were naïve or antiretroviral-experienced and who presented a virological and immunological response to a pre-treatment phase of at least 3 months were recruited. Inclusion criteria were a CD4 count above 350 cells $/ \mu \mathrm{L}$ and HIV viral load (VL) $<50$ copies $/ \mathrm{ml}$ and no evidence for pre-existing drug resistance, such as detectable VL on prior antiretroviral therapy or history of virological failure. The patients were initially randomized in three groups: one group with continuous treatment (CT arm), one group with CD4 guided treatment interruptions (STI arm) with a CD4 threshold of 350 cells $/ \mu \mathrm{L}$ for interruption and re-initiation of antiretroviral therapy (STI:CT ratio 2:1). The third group had a week on therapy followed by a week off therapy. This group was stopped prematurely due to a high frequency of virological failure [18]. For the present study, only patients randomized in the CT and in the STI arms were analysed.

\section{Mental Health and QOL Scales}

To assess mental health and QoL, we used standardized questionnaires which are validated in Thai language: HIVMedical Outcome Study (MOS-HIV) [19], Hospital Anxiety and Depression Scale (HADS) [20], Depression Anxiety Stress Scale (DASS) [21]. These test are not diagnostic tests but screening instruments and do not replace a assessment by a psychiatrist. Patients were asked to complete the validated Thai version of MOS-HIV, DASS and HADS questionnaires every 24 weeks beginning at baseline visit (randomisation). We will present patients' answers at baseline, week 24, week 48 and at the last visit before restarting cART (in the STI arm), named "last time-point visit" in the text.

The present study was performed in accordance with the approval of the ethical committee of the Thai Ministry of Public Health, and the local ethical committees.

\section{HIV Medical Outcome Study (MOS-HIV)}

The MOS-HIV questionnaire measures health related QoL and was derived from the Medical Outcome Study designed to measure QoL in primary care and general patient populations $[19,22,23]$. It is divided in 10 subscores ranging from 0 to 100 (with better quality of the functional area when greater values) which assessed general health, physical functioning, role functioning, social functioning, cognitive functioning, pain, mental health, vitality, health distress, and QoL. In order to simplify data analysis and the interpretation of findings from clinical trials, and across studies, physical and mental health summary scores (PHS and MHS respectively) were calculated. For the PHS score, the physical function, pain, and role function scale scores contributed most strongly. For the MHS score, the mental health, health distress, quality of life, and cognitive function scales contributed most strongly. The vitality, general health and social functioning scales contributed to both factors. The summary scores were transformed to standardized z-scores, with a mean of 50 and a standard deviation of 10 [23]. MOSHIV has been translated in Thai and used in HIV studies in Thai patients $[10,24,25]$. According to several studies [26, 27] the MOS-HIV questionnaire has good internal consistency, with a Cronbach alpha coefficient [28] reported exceeding 0.75. In the current study, the Cronbach alpha coefficient increased between baseline and the last timepoint visit going from 0.71 to 0.94 (0.39-0.64 for role functioning subscale), depending on the subscale studied.

\section{Hospital Anxiety and Depression Scale (HADS)}

The HADS is a short self-assessment questionnaire developed specifically for an internal medicine context in which milder forms of anxiety and depression are more prevalent [29]. The HADS scale is divided in two sections: depression and anxiety. We considered patients to be depressed or anxious when they scored $\geq 11$. Abnormal or borderline abnormal items were considered as "abnormal". According to Savard et al. [30] the HADS has good internal consistency, with a Cronbach alpha coefficient [28] reported of 0.89 . In the present study, we received only aggregated results which did not allow us to measure the Cronbach alpha coefficient in our study.

\section{Depression Anxiety Stress Scale (DASS)}

The DASS is a 42 item self-report inventory that yields 3 factors: depression, anxiety, and stress. This screening and outcome measure reflects the past 7 days, and reliability of the three scales has proven to be adequate [31]. Patients were considered to be depressed, anxious, or stressed when they answered at least one item with "mild" (score $\geq 10$ for depression, score $\geq 8$ for anxiety and score $\geq 15$ for stress). According to Brown et al. [31] the DASS has an adequate internal consistency, with a Cronbach alpha coefficient [28] reported of $0.96,0.89$ and 0.93 for depression, anxiety and stress. In the present study, we received only aggregated results which did not allow us to measure the Cronbach alpha coefficient.

Both the DASS and HADS questionnaires are accepted tools to assess anxiety and depression [23]. The Thai version of both questionnaires has been validated for use in the Thai population. 


\section{Statistical Analysis}

Both treatment arms were compared at baseline on demographic and clinical characteristics, HADS, DASS, mean PHS and mean MHS scores, using chi-square test of independence or Fisher's exact test for categorical variables and Student- $t$ tests or Mann-Whitney non-parametric test for continuous variables. Patients who filled out the initial questionnaire but not the last one were compared to those who filled out initial and final questionnaires on demographic and clinical characteristics and QoL scores. McNemar test was performed to assess the paired-proportion for abnormal score using HADS and DASS between baseline and last time-point visit. We considered abnormal scores as a combination of abnormal and borderline scores using HADS scale. For DASS, we stratified abnormal scores in mild to moderate and severe to extremely severe scores.

HADS, DASS and MOS scores were analyzed from baseline to last time-point visit. A mixed between-within subjects analysis of variance (ANOVA) was used to test the impact of the arm of randomization on PHS and MHS across four time points $(0,24,48$ weeks and last time-point visit).

All analyses were done with SPSS version 11.0 (SPSS Inc., Chicago, Illinois, USA). Two-sided p-value $<0.05$ was considered statistically significant.

\section{RESULTS}

A total of 379 Thai patients were enrolled in STACCATO from January 2002, and 251 were included in the present substudy from March 2003. Among them, all patients had answered the HADS and DASS questionnaires at baseline, and $207(82.5 \%)$ at the last time-point visit. For MOS-HIV, 241 (96\%) patients answered the questionnaire at baseline and 133 patients at the last time-point visit (55.2\%).

Baseline characteristics of patients who answered to HADS/DASS and MOS-HIV questionnaires are shown in Table 1.

Patients who only filled out the initial questionnaire on HADS and DASS $(n=43,17.1 \%)$ were compared to those who filled out both initial and final questionnaires $(n=208)$. They were distributed equally in both treatment arms $(p=0.46)$, had similar demographical characteristics (age $\mathrm{p}=0.86$, sex $\mathrm{p}=0.99$ ) and clinical characteristics (CDC stage $\mathrm{p}=0.69$, proportion of $\mathrm{MSM} \mathrm{p}=0.19$, proportion of intravenous drug users $\mathrm{p}=0.82$, proportion of heterosexual risks $\mathrm{p}=0.25$, mean HIV-RNA before cART $\mathrm{p}=0.86$, mean CD4 count before cART $\mathrm{p}=0.57)$. Regarding distribution of anxiety, depression or stress from DASS scale, both groups had similar distributions ( $\mathrm{p}=0.59,0.99$ and 0.68 respectively) at baseline. Distribution of depression and anxiety using HADS scale at baseline were also similar between both groups ( $\mathrm{p}=0.99$ and 0.13 respectively).

Patients who filled out the initial questionnaire on MOSHIV ( $n=51,21.2 \%$ ) were compared to those who filled out both initial and final questionnaires $(n=190)$. They were distributed equally in both treatment arms $(p=0.43)$, had similar demographical characteristics (age $p=0.59$, sex

Table 1. Baseline Characteristics of Patients Assessed with DASS/HADS and MOS-HIV Questionnaires

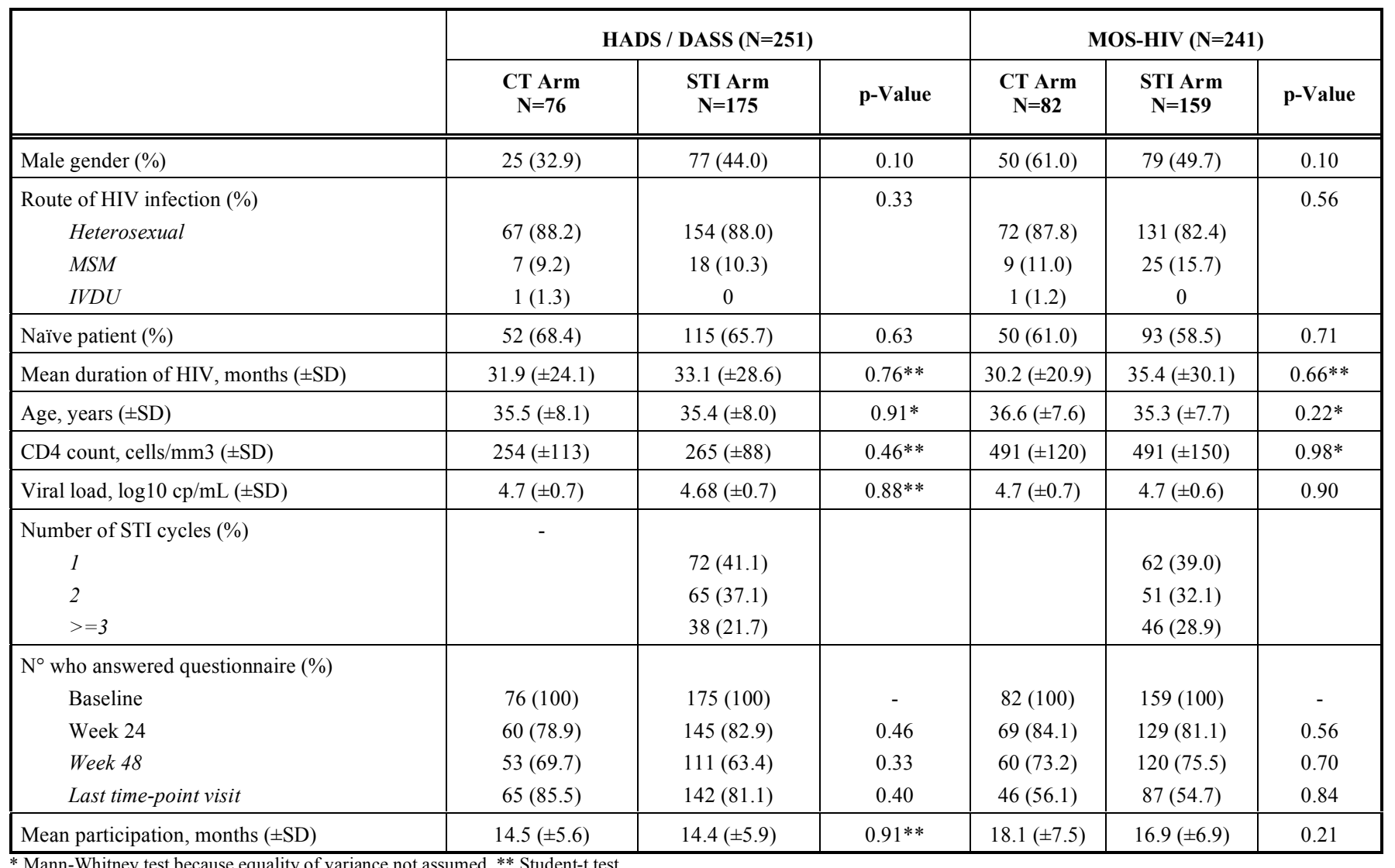


$\mathrm{p}=0.16$ ) and clinical characteristics (CDC stage $\mathrm{p}=0.90$, proportion of MSM tends to higher among patients who filled both periods $\mathrm{p}=0.06$, proportion of intra-venous drug users $p=0.99$, proportion of heterosexual risks $p=0.19$, mean HIV-RNA before cART $\mathrm{p}=0.24$, mean CD4 count before cART $\mathrm{p}=0.53)$. Regarding mean PHS and MHS scores at baseline, there was no significant difference $(p=0.27$ and 0.72 respectively).

Table 2 presents the prevalence of anxiety or depression using HADS and the prevalence of mild-moderate and severe to extremely severe anxiety, depression and stress using DASS in each arm, at baseline; there is no difference between arms (all p-value $>0.05$ ). We show that $2.6 \%$ of patients randomized in the CT arm and $0.6 \%$ in the STI arm had a combination of anxiety and depression at baseline with the HADS scale $(\mathrm{p}=0.22)$. With the DASS scale, $3.9 \%$ of patients included in the CT arm and $1.2 \%$ of patients included in the STI arm had a combination of mild to moderate anxiety and depression ( $\mathrm{p}=0.16) ; 2.6 \%$ of patients randomized in the CT arm versus none in the STI arm had a combination of severe anxiety and depression at baseline $(\mathrm{p}=0.09)$.

Table 3 shows the comparison between arms of anxiety, depression and stress from baseline to last time-point visit (65 patients in the CT arm and 142 in the STI arm). We found a significantly lower proportion of mild to moderate stress between both time-points in the STI arm $(\mathrm{p}=0.004)$ and a significantly lower proportion of severe to extremely severe anxiety between both time-points in the STI arm $(p=0.008)$. The comparison of abnormal values regarding anxiety, depression and stress between baseline and last time-point visit was similar inside CT arm whatever HADS or DASS scales; nonetheless there was a tendency for higher severe stress in the CT arm at last time-point visit compared to baseline $(p=0.07)$. We found a significant higher proportion of depression at last time-point visit compared to baseline in the STI arm using the HADS scale (McNemar test, $\mathrm{p}=0.004$ ) while the difference was not statistically different using DASS scale. With the HADS, we found that anxiety was the same between baseline and last time-point visit in the STI arm while with the DASS, the proportion of severe anxiety was significantly decreased in the STI arm at last time-point visit $(\mathrm{p}=0.008)$ but not for mild to moderate anxiety $(p=0.75)$. Regarding stress, the proportion of patients who were mildly to moderately stressed at baseline decreased significantly at last time-point visit in the STI arm $(\mathrm{p}=0.04)$ but the proportion of severely stressed patients was similar between both time-points within the STI arm $(\mathrm{p}=0.32)$.

In the MOS-HIV questionnaire exploring the QoL at baseline, the mean MHS score was significantly lower in the STI arm compared to CT arm (49.1 \pm 8.3 versus $51.7 \pm 7.6$, $\mathrm{p}=0.02$ ) and the PHS score was similar between arms (54.4 \pm 6.3 versus $53.8 \pm 6.6, \mathrm{p}=0.53)$. The variation of the PHS and MHS scores from baseline to last time-point visit (with their 95\% confidence interval) is presented in Fig. (1). Regarding the PHS, mixed between-within subjects ANOVA showed that there was no significant interaction between the four time-points and the randomization arm $(p=0.74$; partial eta squared $=0.01$ ). There was neither change of PHS across the four time-points $(\mathrm{p}=0.15)$ nor between the two arms of randomization $(\mathrm{p}=0.45)$ (Fig. 1b). Regarding the MHS, we

Table 2. Proportion of Anxiety, Depression and Stress Using HADS and DASS Scales between CT and STI Arms at Baseline

\begin{tabular}{|c|c|c|c|c|c|}
\hline & \multicolumn{2}{|c|}{$\begin{array}{c}\text { CT Arm } \\
(\mathrm{N}=76)\end{array}$} & \multicolumn{2}{|c|}{$\begin{array}{c}\text { STI Arm } \\
(N=175)\end{array}$} & \multirow{2}{*}{ p-Value } \\
\hline & $\mathbf{N}$ & $\%$ & $\mathbf{N}$ & $\%$ & \\
\hline \multicolumn{6}{|l|}{ HADS scale } \\
\hline Anxiety & 12 & 15.8 & 29 & 16.6 & 0.88 \\
\hline Depression & 8 & 10.5 & 10 & 5.7 & 0.17 \\
\hline Combination of anxiety and depression & 2 & 2.6 & 1 & 0.6 & $0.22 *$ \\
\hline \multicolumn{6}{|l|}{ DASS scale } \\
\hline \multicolumn{6}{|l|}{ Anxiety } \\
\hline Mild-moderate & 25 & 32.9 & 63 & 36.0 & 0.64 \\
\hline Severe-extremely severe & 5 & 6.6 & 19 & 10.6 & 0.29 \\
\hline \multicolumn{6}{|l|}{ Depression } \\
\hline Mild-moderate & 7 & 9.2 & 15 & 8.6 & 0.87 \\
\hline Severe-extremely severe & 3 & 3.9 & 2 & 1.1 & 0.16 \\
\hline \multicolumn{6}{|l|}{ Combination of anxiety and depression } \\
\hline Mild-moderate & 3 & 3.9 & 2 & 1.2 & $0.16^{*}$ \\
\hline Severe-extremely severe & 2 & 2.6 & - & - & $0.09 *$ \\
\hline \multicolumn{6}{|l|}{ Stress } \\
\hline Mild-moderate & 33 & 43.4 & 74 & 42.3 & 0.87 \\
\hline Severe-extremely severe & 4 & 5.3 & 8 & 4.6 & $0.76^{*}$ \\
\hline
\end{tabular}

\footnotetext{
* Fisher's exact test.
} 
Table 3. Comparison of Depression, Anxiety and Stress Prevalence between Baseline and Last Time-Point Visit Scored by HADS or DASS between Both Randomization Arms

\begin{tabular}{|c|c|c|c|c|c|c|}
\hline \multirow[t]{2}{*}{ Arms } & \multicolumn{2}{|c|}{ Anxiety } & \multicolumn{2}{|c|}{ Depression } & \multicolumn{2}{|c|}{ Stress } \\
\hline & $\underset{(n=65)}{C T}$ & $\underset{(n=142)}{\text { STI }}$ & $\begin{array}{c}\mathrm{CT} \\
(\mathrm{n}=65)\end{array}$ & $\underset{(n=142)}{\text { STI }}$ & $\begin{array}{c}\text { CT } \\
(n=65)\end{array}$ & $\begin{array}{c}\text { STI } \\
(n=142)\end{array}$ \\
\hline \multicolumn{7}{|l|}{ HADS scale (\%) } \\
\hline \multicolumn{7}{|l|}{ Abnormal values* } \\
\hline Baseline & $12(18.5)$ & $23(16.2)$ & $8(12.3)$ & $6(4.2)$ & - & - \\
\hline Last time-point visit & $13(20.0)$ & $28(19.7)$ & $8(12.3)$ & $18(12.7)$ & - & - \\
\hline$p$-value & 0.99 & 0.49 & 0.99 & 0.004 & - & - \\
\hline \multicolumn{7}{|l|}{ DASS scale (\%) } \\
\hline \multicolumn{7}{|l|}{ Mild to moderate } \\
\hline Baseline & $22(33.8)$ & $48(33.8)$ & $7(10.8)$ & $9(6.3)$ & $20(30.8)$ & $42(29.6)$ \\
\hline Last time-point visit & $20(30.8)$ & $46(32.4)$ & $5(7.7)$ & $8(5.6)$ & $12(18.5)$ & $29(20.4)$ \\
\hline$p$-value & 0.67 & 0.75 & 0.53 & 0.78 & 0.07 & 0.04 \\
\hline \multicolumn{7}{|l|}{ Severe to extremely severe } \\
\hline Baseline & $5(7.7)$ & $15(10.6)$ & $1(1.5)$ & $1(0.7)$ & $2(3.1)$ & $3(2.1)$ \\
\hline Last time-point visit & $2(3.1)$ & $5(3.5)$ & 0 & 0 & $4(6.2)$ & $5(3.5)$ \\
\hline$p$-value & 0.26 & 0.008 & 0.32 & 0.32 & 0.32 & 0.32 \\
\hline
\end{tabular}

* Borderline and abnormal items were considered abnormal. Numbers of patients changed depending on the subscale.

showed that there was no significant interaction between the four time-points and the randomization arm $(p=0.43)$. There was a substantial main effect across time-points $(\mathrm{p}=0.001$, partial eta squared $=0.128$ corresponding to a moderate to high effect size) showing an increase of mental health across the 4 time-points. The main effect comparing the randomization arm was not significant $(\mathrm{p}=0.17$, partial eta squared $=0.014)$, suggesting no difference in the evaluation of mental health between both randomization arms (Fig. 1a). When we restricted our analysis to STI arm taking into account the number of STI cycles (divided in three categories: 1, 2 or $\geq 3$ STI cycles), mixed between-within subjects ANOVA assessing physical health showed that there was no significant interaction between the four timepoints and the number of STI cycles $(p=0.71)$, PHS was stable across the four time-points $(p=0.64)$ and there was no significant change regarding the number of STI cycles $(p=0.27)$. The model that assessed mental health showed that there was no significant interaction between time and the number of STI cycles $(p=0.57)$ but that there was a significant increase of MHS across the four time-points $(p=0.007)$ without a difference regarding the number of STI cycles $(\mathrm{p}=0.30)$.

We used a multivariate linear model to explore the association between PHS and MHS scores at baseline and final visit with various covariates. At baseline, MHS was greater among patients over 44 years $(p=0.08)$, among patients infected through heterosexual intercourse $(\mathrm{p}=0.8)$, when CD4 count was above 500 cells/ $\mu \mathrm{L}(\mathrm{p}=0.009)$ and increased with weight $(\mathrm{p}=0.09)$. PHS at baseline was lower among patients infected through heterosexual intercourse $(p=0.04)$ and among patients screened for HIV for more than 6 months $(\mathrm{p}=0.12)$. For the last visit, MHS was lower among patients infected through heterosexual intercourse $(p=0.01)$, among patients randomized in the CD4 guided group $(p=0.04)$ and among patients who lost more than $5 \mathrm{~kg}$ from baseline $(\mathrm{p}=0.001)$. For PHS, the score was lower in women $(p=0.06)$, if patients had triglycerides above $200 \mathrm{mg} / \mathrm{dl}$ $(p=0.05)$, if LDL-cholesterol was greater than $140 \mathrm{mg} / \mathrm{dl}$ $(p=0.09)$ and if patients lost more than $5 \mathrm{~kg}$ from baseline $(p=0.06)$. Globally, multivariate linear models did not fit perfectly with adjusted covariates $\left(R^{2}<0.10\right.$ for the 4 models).

\section{DISCUSSION}

Since the advent of cART, morbidity and mortality of HIV-infections have drastically decreased in industrialized countries [32]. HIV infection has become a chronic disease with a quasi normal life expectancy. In the cART era, QoL now represents a challenge in the care management of HIVinfected patients. Stigmatizing side effects such as lipodystrophy [33] have to be taken into account choosing antiretroviral drugs. The strategy of STI was proposed as an alternative to continuous cART in patients with moderate immunosuppression (CD4 count above 350 cells $/ \mu \mathrm{L}$ ) but since the publication of the SMART trial's results, it is not recommended at a patient-population level [14]. Indeed with few exceptions [34, 35], the results of STI on QoL were disappointing [36-38]. In some studies its impact on general health perception was even negative $[14,39]$. In accordance, our results showed no benefit of STI on mental health and QoL. But what appeared is that cART by itself has a positive effect on mental health. Even in patients randomized to the STI arm, and who for unknown reasons had MHS scores which were significantly lower at baseline, a significant increase of MHS scores across study time-points was seen. This suggests that they have corrected their MHS score during STACCATO trial while on continuous treatment. At last time-point visit they reached the same scores as patients randomized in the $\mathrm{CT}$ arm at baseline. We also showed a tendency to lower proportions of anxiety and stress between baseline and last time-point visit among patients randomized 
(a)
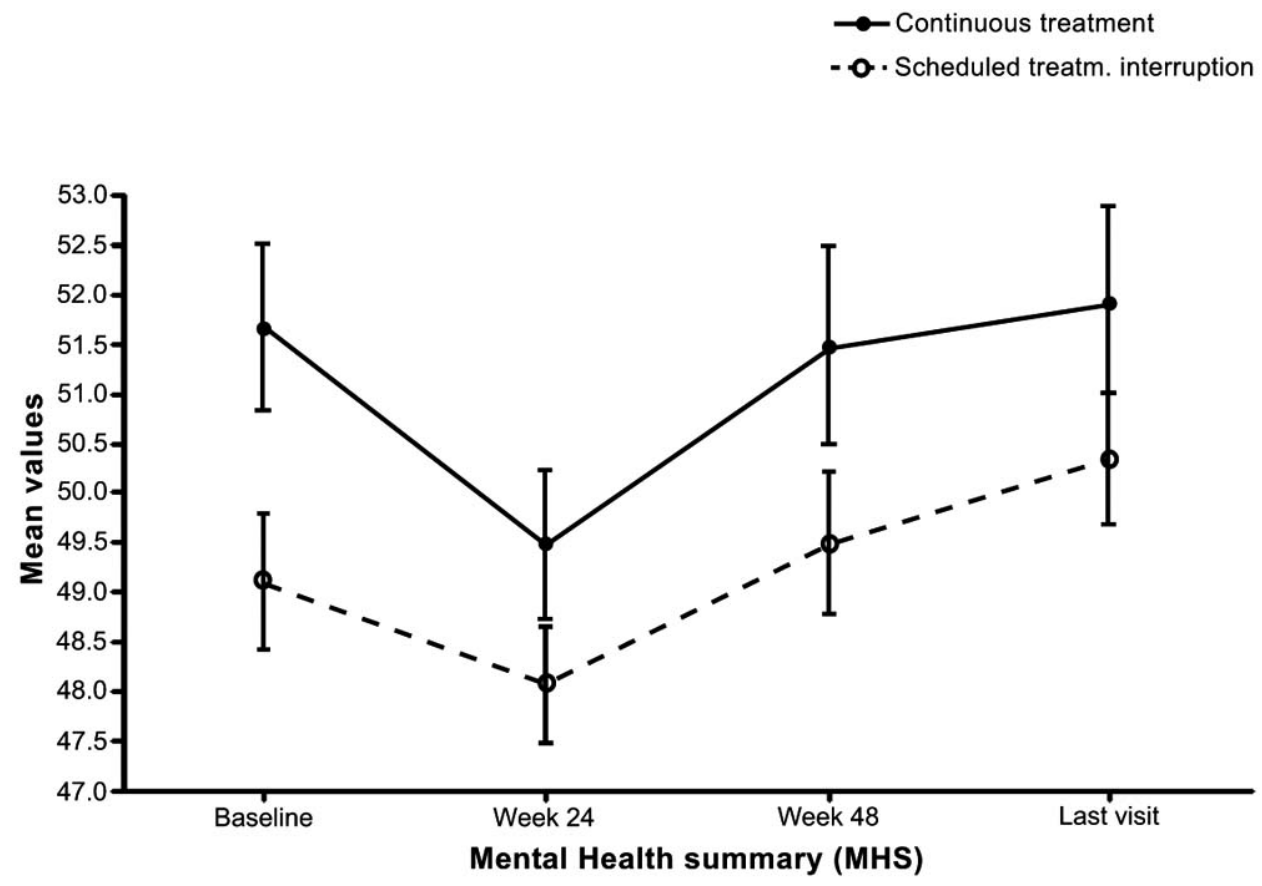

(b)

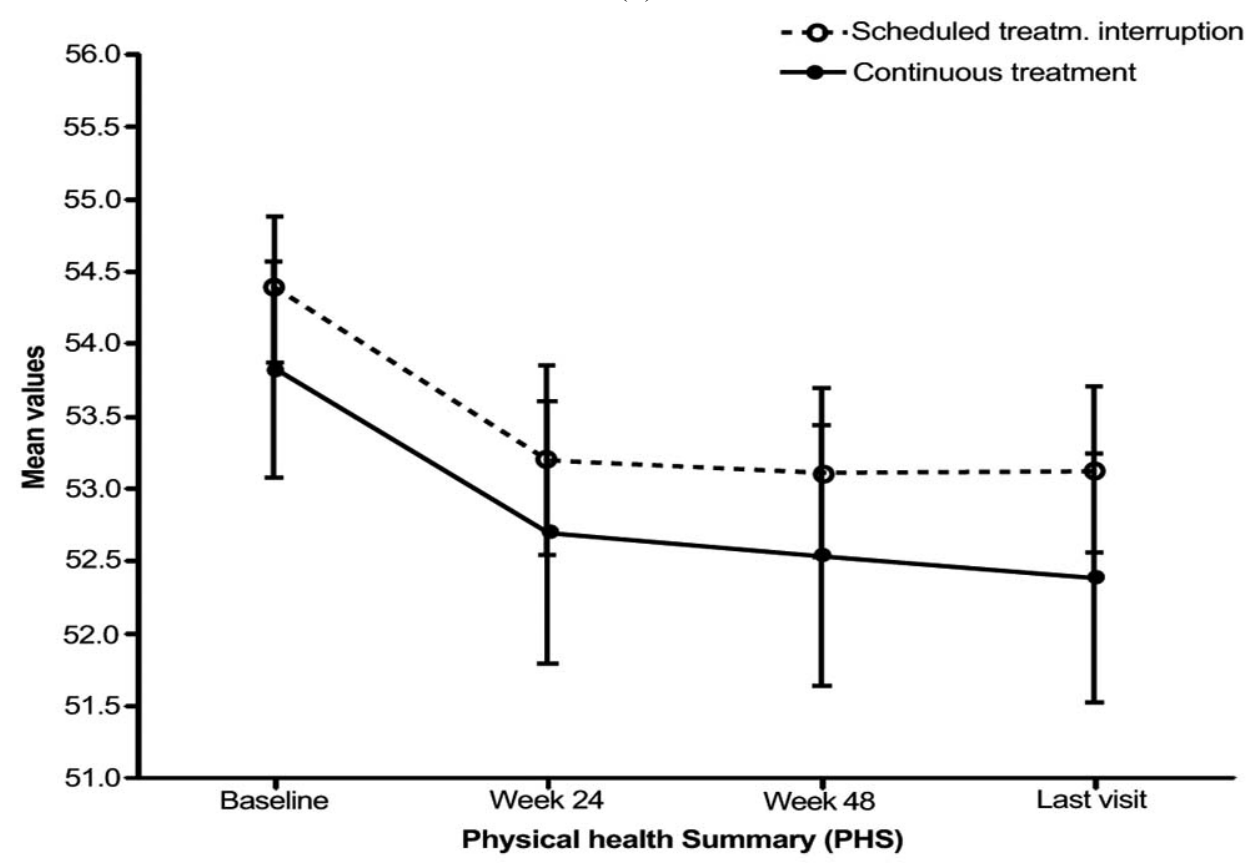

Fig. (1). Line graphs of mean scores with their 95\% confidence intervals for the Mental Health summary (MHS) (1a) and Physical Health Summary (PHS) (1b) by arm and across the four time-points. MHS and PHS are T-scored with a mean of 50 and a standard deviation of 10. Last visit corresponds to last time-point visit.

in the STI arm. Regarding depression, we found contradictory results. A higher rate of depression at the last time-point visit compared to baseline among patients randomized in the STI arm, but not in the continuous arm.

For our investigation we used the well-known and validated questionnaires HADS, DASS and MOS-HIV. MOS-HIV also measures fatigue, a important variable affecting QOL in HIV-HCV co-infection [40]. These questionnaires are in fact measurement tools that have coefficients of variation comparable to CD4 counts [41, 42]. We showed that the number of patients who answered the questionnaires was different and we explained this disparity by the fact that in Thailand the STACCATO trial was organized in seven centres with at least seven clinical investigators who did not always adhere to the protocol. The focus was on mental health, anxiety, stress and depression in particular. Most of our patients lived in the Bangkok metropolitan area with a lifetime prevalence of anxiety 
disorders in the general population of $10.2 \%$, and $19.9 \%$ depression [43]. In the literature prevalence of depression and anxiety among people living with HIV-infection and treated with antiretroviral therapy varies from $4 \%$ to $51 \%$ [44-49]. This wide range is due to the multiplicity of settings evaluated, questionnaires used and to the diversity of mental disorders studied, from mild to severe. In our study prevalence of severe depression measured at baseline by DASS was $2.0 \%$, which is low. If mild and moderate forms are included, depressive symptoms were seen in $8.8 \%$ and anxiety in $35.1 \%$. Mental health and QoL were found similar in the STI arm compared with CT. The number of STI did not modify the results at the different questionnaires as we could expect.

We should address some limitations to our study. First only $63.6-66.2 \%$ of all enrolled Thai patients answered the MOS-HIV and HADS/DASS questionnaires, which is few. To rule out biases, we compared patients who only filled out the initial questionnaire on HADS and DASS to those who filled out both initial and final questionnaires. They were distributed equally within both arms and had similar characteristics making a selection unlikely. We should admit a potential selection bias because all participants were volunteers. This could explain the relatively low prevalence of anxiety, depression or stress at baseline. Probably they are not representative of the entire HIV-infected population in whom mental disorders are more frequent [1-3]. Also it must be emphasized that the screening tests we used are not equivalent to an assessment by a psychiatrist. Finally time within randomized phase of STACCATO was between 14.4 and 16.5 months and most of the patients had less than 2 treatment interruptions which could also explained the relatively low prevalence of mental health disorders emerging during the study. But even in this motivated group the positive effect of cART was evident.

In a previous publication, we showed that $\mathrm{CD} 4$ guided STI led to less drug toxicity, particularly neuropathy, but also to more HIV-associated symptoms like oral candidiasis or acute retroviral syndrome [17]. Most of the drug toxicity was associated with stavudine, a drug still widely used in resource limited settings. Even with this relatively toxic regimen, QoL improved with continuous cART, not with STI. Further, HIV infection is known to affect the central nervous system [50]. Thus uncontrolled viremia can have an impact on mental health. The vast majority of our patients started antiretroviral therapy approximately 6 months before enrollment into STACCATO. This relatively short duration of treatment must be taken into account when interpreting our findings, as treatment fatigue might be less pronounced than in other trials with longer duration of cART. On the other hand, trials with a longer previous time on cART failed to show a benefit of STI on QoL as well [14, 36-39].

In summary, continuous cART has a beneficial effect on mental health in Thai patients with chronic HIV infection.
The HIV Netherlands Australia Thailand Research Collaboration, Bangkok, Thailand: Praphan Phanuphak, Sukontha Saenawat, Saijai Wicharuk, Siriporn Nonenoy, Natnipa Wannachai, Sineenart Chautrakarn, Theshinee Chuenyam, Thanthip Nuchapong, Apicha Mahanontharit, Jongkol Sankonte, Bucha Piyavong, Vantanit Piroj, Napawan Seekaow, Wipawan Karakte and Sasiwimol Ubolyam.

Bamrasnaradura Institute, Nonthaburi, Thailand: Suchittra Putthwong, Sirirat Liganonsakul, Wattana Sanchiem, Pongpan Boonchoo, Malee Suannum.

Khon Kaen University, Khon Kaen, Thailand: Parichat Bunyaprawit, Ratthanant Kaewmarg.

Sanpatong Hospital, Chiang Mai, Thailand Phitsanu Raksakulkarn, Yaowaluk Penglimoon.

Buddhachinnaraj Hospital, Phitsanulok: Sopha Khongsawad.

\section{TRIAL REGISTRATION}

The STACCATO trial is registered at ClinicalTrials.gov with the identifier NCT00113126.

\section{ACKNOWLEDGEMENTS}

The Swiss HIV Cohort study provided logistic support for Staccato. The antiretrovirals were provided at no cost by Roche (saquinavir), Abbott (ritonavir) and Gilead (tenofovir). Bristol Myers Squibb provided stavudine and didanosine at a reduced price.

\section{REFERENCES}

[1] Bing EG, Burnam MA, Longshore D, et al. Psychiatric disorders and drug use among human immunodeficiency virus-infected adults in the United States. Arch Gen Psychiatry 2001; 58(8): 721-28.

[2] Atkinson JH, Grant I, Kennedy CJ, et al. Prevalence of psychiatric disorders among men infected with human immunodeficiency virus. A controlled study. Arch Gen Psychiatry 1988; 45(9): 859-64.

[3] Lyketsos CG, Hanson A, Fishman M, McHugh PR, Treisman GJ. Screening for psychiatric morbidity in a medical outpatient clinic for HIV infection: the need for a psychiatric presence. Int J Psychiatry Med 1994; 24(2): 103-13.

[4] Hartzell JD, Janke IE, Weintrob AC. Impact of depression on HIV outcomes in the HAART era. J Antimicrob Chemother 2008; 62(2): 246-55.

[5] Zinkernagel C, Taffe P, Rickenbach M, et al. Importance of mental health assessment in HIV-infected outpatients. J Acquir Immune Defic Syndr 2001; 28(3): 240-49.

[6] Benson CA.Structured treatment interruption in HIV infection. AIDS Read 2001; 11(2): 99-102.

[7] Hirschel B. Planned interruptions of anti-HIV treatment. Lancet Infect Dis 2001; 1(1): 53-9.

[8] Lori F, Maserati R, Foli A, et al. Structured treatment interruptions to control HIV-1 infection. Lancet 2000; 355(9200): 287-88.

[9] Zhou J, Kumarasamy N, Ditangco R, et al. The TREAT Asia HIV Observational Database: baseline and retrospective data. J Acquir Immune Defic Syndr 2005; 38(2): 174-79.

[10] Ananworanich J, Siangphoe U, Hill A, et al. Highly active antiretroviral therapy (HAART) retreatment in patients on CD4guided therapy achieved similar virologic suppression compared with patients on continuous HAART: the HIV Netherlands Australia Thailand Research Collaboration 001.4 study. J Acquir Immune Defic Syndr 2005; 39(5): 523-29.

\section{THE STACCATO STUDY GROUP}


[12] Friis-Moller N, Sabin CA, Weber R, et al. Combination antiretroviral therapy and the risk of myocardial infarction. N Engl J Med 2003; 349(21): 1993-2003.

[13] Friis-Moller N, Weber R, Reiss P, et al. Cardiovascular disease risk factors in HIV patients--association with antiretroviral therapy. Results from the DAD study. AIDS 2003; 17(8): 1179-93.

[14] El-Sadr WM, Lundgren JD, Neaton JD, et al. CD4+ count-guided interruption of antiretroviral treatment. N Engl J Med 2006; 355(22): 2283-96.

[15] Ananworanich J, Hirschel B. Intermittent therapy for the treatment of chronic HIV infection. AIDS 2007; 21(2): 123-34.

[16] Nüesch R, Hirschel B. Treatment interruption for convenience, cost cutting and toxicity sparing. Curr Opin HIV AIDS 2007; 2: 31-38.

[17] Ananworanich J, Gayet-Ageron A, Le Braz M, et al. CD4-guided scheduled treatment interruptions compared with continuous therapy for patients infected with HIV-1: results of the Staccato randomised trial. Lancet 2006; 368(9534): 459-65.

[18] Ananworanich J, Nuesch R, Le Braz M, et al. Failures of 1 week on, 1 week off antiretroviral therapies in a randomized trial. AIDS 2003; 17(15): F33-37.

[19] Wu AW, Revicki DA, Jacobson D, Malitz FE. Evidence for reliability, validity and usefulness of the Medical Outcomes Study HIV Health Survey (MOS-HIV). Qual Life Res 1997; 6(6): 481-93.

[20] Snaith RP, Zigmond AS. The hospital anxiety and depression scale. Br Med J (Clin Res Ed). 1986; 292(6516): 344.

[21] Lovibond SH, Lovibond PF. Depression anxiety stress scale. 2008. Available from: http://www2.psy.unsw.edu.au/groups/dass/.

[22] Wu AW. Quality of life assessment comes of age in the era of highly active antiretroviral therapy. AIDS 2000; 14(10): 1449-51.

[23] Wu AW, Hays RD, Kelly S, Malitz F, Bozzette SA. Applications of the Medical Outcomes Study health-related quality of life measures in HIV/AIDS. Qual Life Res 1997; 6(6): 531-54.

[24] Ichikawa M, Natpratan C. Quality of life among people living with HIV/AIDS in northern Thailand: MOS-HIV Health Survey. Qual Life Res 2004; 13(3): 601-10.

[25] Cardiello PG, Hassink E, Ananworanich J, et al. A prospective, randomized trial of structured treatment interruption for patients with chronic HIV type 1 infection. Clin Infect Dis 2005; 40(4): 594-600.

[26] Burgess A, Dayer M, Catalan J, Hawkins D, Gazzard B. The reliability and validity of two HIV-specific health-related Quality-ofLife measures: a preliminary analysis. AIDS 1993; 7(7): 1001-008.

[27] Carretero MD, Burgess AP, Soler P, Soler M, Catalan J. Reliability and validity of an HIV-specific health-related quality-of-life measure for use with injecting drug users. AIDS 1996; 10(14): 1699-705.

[28] Cronbach L. Coefficient alpha and the internal structure of test. Psychometrika 1951; 16: 297-334

[29] Zigmond AS, Snaith RP. The hospital anxiety and depression scale. Acta Psychiatr Scand 1983; 67(6): 361-70.

[30] Savard J, Laberge B, Gauthier JG, Ivers H, Bergeron MG. Evaluating anxiety and depression in HIV-infected patients. J Pers Assess 1998; 71(3): 349-67.

[31] Brown TA, Chorpita BF, Korotitsch W, Barlow DH. Psychometric properties of the Depression Anxiety Stress Scales (DASS) in clinical samples. Behav Res Ther 1997; 35(1): 79-89.

[32] Palella FJ, Delaney KM, Moorman AC, et al. Declining morbidity and mortality among patients with advanced human immunodeficiency virus infection. HIV Outpatient Study Investigators. N Engl J Med 1998; 338(13): 853-60.

[33] Guaraldi G, Murri R, Orlando G, et al. Severity of lipodystrophy is associated with decreased health-related quality of life. AIDS Patient Care STDS 2008; 22(7): 577-85.

[34] Cohen CJ, Colson AE, Sheble-Hall AG, McLaughlin KA, Morse GD. Pilot study of a novel short-cycle antiretroviral treatment interruption strategy: 48-week results of the five-days-on, two-daysoff (FOTO) study. HIV Clin Trials 2007; 8(1): p. 19-23.
[35] Ruiz L, Paredes R, Gómez G, et al. Antiretroviral therapy interruption guided by CD4 cell counts and plasma HIV-1 RNA levels in chronically HIV-1-infected patients. AIDS 2007; 21(2): 16978.

[36] Marchou B, Tangre P, Charreau I, et al. Intermittent antiretroviral therapy in patients with controlled HIV infection. AIDS 2007; 21(4): 457-66.

[37] Maserati R, Foli A, Tomasoni L, et al. Effects of structured treatment interruptions on metabolic, anthropometric, immunologic, and quality of life outcomes in HIV-positive adults on HAART. Curr HIV Res 2007; 5(3): 337-43.

[38] Palmisano L, Giuliano M, Bucciardini R, et al. Determinants of virologic and immunologic outcomes in chronically HIV-Infected subjects undergoing repeated treatment interruptions: the istituto superiore di sanita-pulsed antiretroviral therapy (ISS-PART) study. J Acquir Immune Defic Syndr 2007; 46(1): 39-47.

[39] Burman W, Grund B, Roediger MP, Friedland G, Darbyshire J, Wu $\mathrm{AW}$. The impact of episodic CD4 cell count-guided antiretroviral therapy on quality of life. J Acquir Immune Defic Syndr 2007; 25: 25.

[40] Marcellin F, Preau M, Ravaux I, Dellamonica P, Spire B, Carrieri MP. Self-reported fatigue and depressive symptoms as main indicators of the quality of life (QOL) of patients living with HIV and Hepatitis C: implications for clinical management and future research. HIV Clin Trials 2007; 8(5): 320-27.

[41] Deyo RA, Carter WB. Strategies for improving and expanding the application of health status measures in clinical settings. A researcher-developer viewpoint. Med Care 1992; 30(5 Suppl): MS176-186; discussion MS196-209.

[42] Hughes MD, Stein DS, Gundacker HM, Valentine FT, Phair JP, Volberding PA. Within-subject variation in CD4 lymphocyte count in asymptomatic human immunodeficiency virus infection: implications for patient monitoring. J Infect Dis 1994; 169(1): 28-36.

[43] Thavichachart N, Intoh P, Thavichachart T, et al. Epidemiologica survey of mental disorders and knowledge attitude practice upon mental health among people in Bangkok Metropolis. J Med Assoc Thai 2001; 84(Suppl 1): S118-26.

[44] Lima VD, Geller J, Bangsberg D R, et al. The effect of adherence on the association between depressive symptoms and mortality among HIV-infected individuals first initiating HAART. AIDS 2007; 21(9): 1175-83.

[45] Alciati A, Starace F, Scaramelli B, et al. Has there been a decrease in the prevalence of mood disorders in HIV-seropositive individuals since the introduction of combination therapy? Eur Psychiatry 2001; 16(8): 491-96.

[46] Rabkin, JG, Ferrando SJ, Lin SH, Sewell M, McElhiney M. Psychological effects of HAART: a 2-year study. Psychosom Med 2000; 62(3): 413-22.

[47] Poupard M, Ngom Gueye N F, Thiam D, et al. Quality of life and depression among HIV-infected patients receiving efavirenz- or protease inhibitor-based therapy in Senegal. HIV Med 2007; 8(2): 925.

[48] Ammassari A, Antinori A, Aloisi MS, et al. Depressive symptoms, neurocognitive impairment, and adherence to highly active antiretroviral therapy among HIV-infected persons. Psychosomatics 2004; 45(5): 394-402

[49] Starace F, Bartoli L, Aloisi MS, et al. Cognitive and affective disorders associated to HIV infection in the HAART era: findings from the NeuroICONA study. Cognitive impairment and depression in HIV/AIDS. The NeuroICONA study. Acta Psychiatr Scand 2002; 106(1): 20-6.

[50] Scaravilli F, Bazille C, Gray F. Neuropathologic contributions to understanding AIDS and the central nervous system. Brain Pathol 2007; 17(2): 197-208. 